\title{
Severity of Polyposis and Severity of Lund-McKay scoring
}

\section{Authors: I Robu, A Ojo, E Prades, O Tkachuk, Z Hammad ENT Department Glan Clwyd Hospital, Wales, United Kingdom}

Background:

Lund-Mackay score system is widely used and while not having significant relationship with the symptoms of the patients (SNOT-22) it provides complementary information of interest for diagnosis and treatment of CRS with polyposis. The aim of this study is to demonstrate the relationship between degree of polyposis and degree of L-M score as previously demonstrated in a reference study (Otolaryngology-Head and Neck Surgery (2007) 137, 555561)
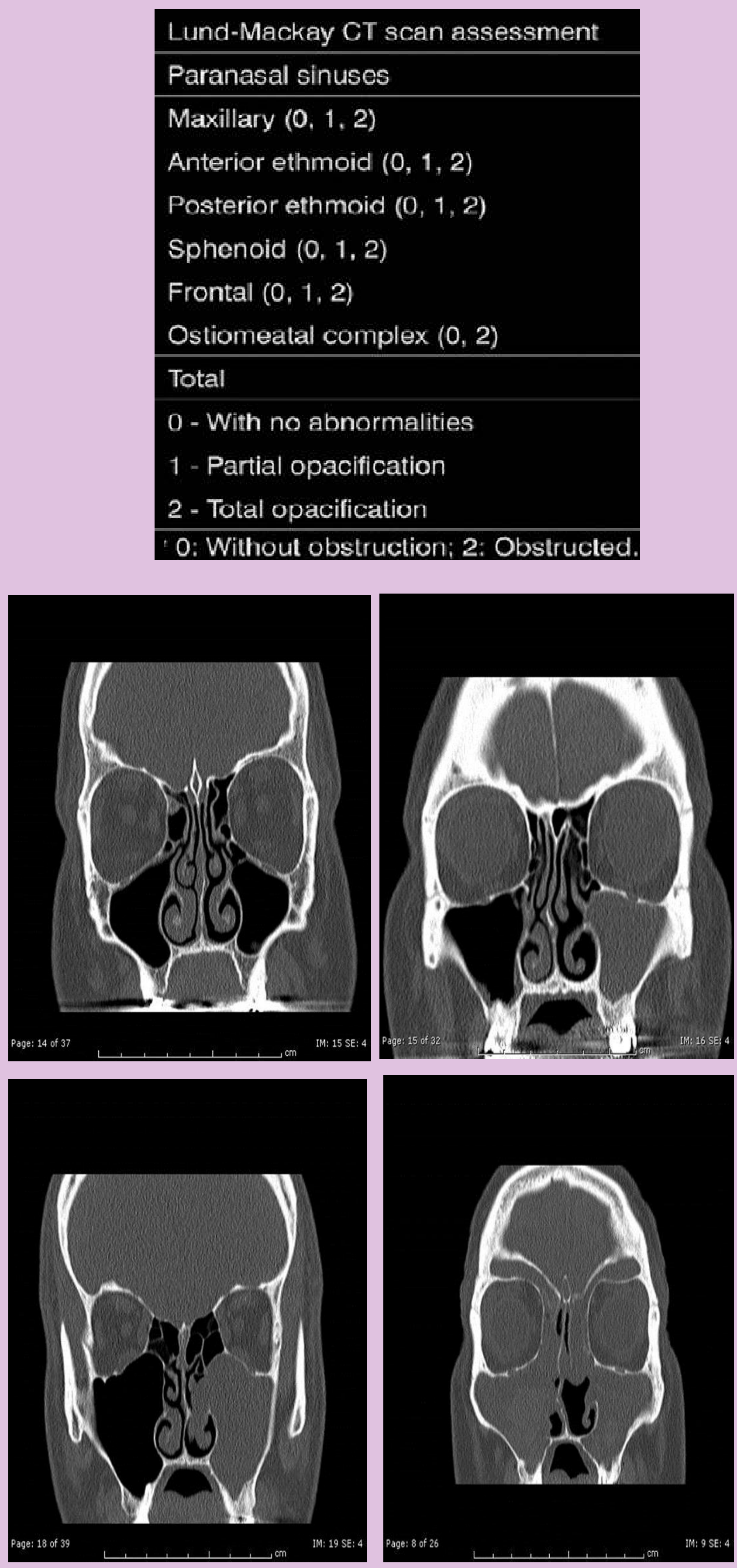

Methods:

100 cases with Bilateral Polyposis that received FESS surgery in our ENT department between 2011 and 2015 from 6 different Consultants were reviewed. One way Anova and means for the L-M score with $\mathrm{Cl} 195 \%$ were performed for the right side scorings.

Results: No significant relationship appeared in our group of patients $(\mathrm{P}=0.12)$. Mean for polyposis grade I was 7.5 (IC95\%: 6.6 to 8.4 ), grade II was 8.6 (IC95\%: 7.9 to 9.4 ) and grade III was 8.9 (IC95\%: 7.7 to 10.1). See figure $X$.

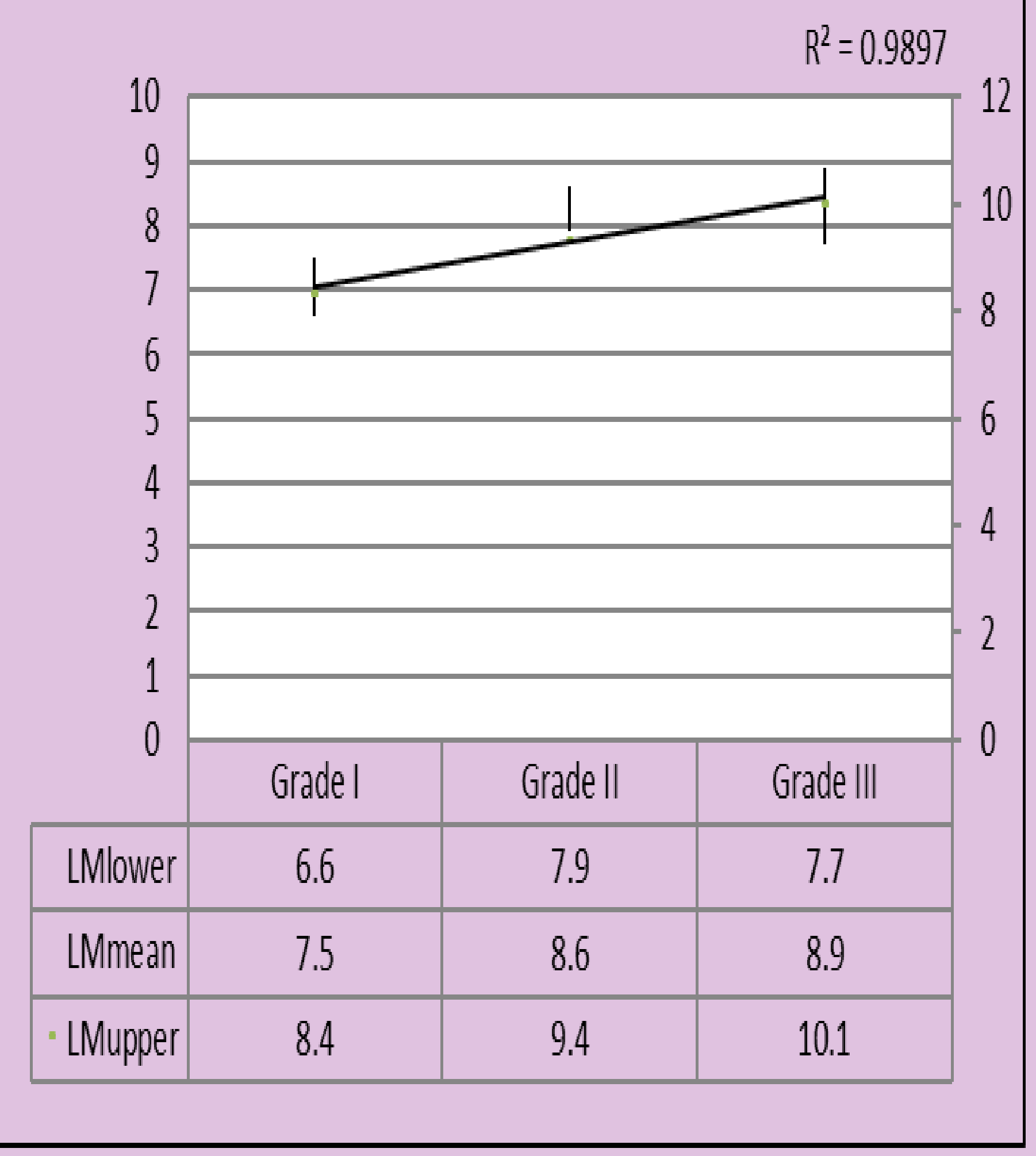

Fig X. Relationship between Grade of endoscopic Polyposis and Lund Mackay score for the right paranasal sinuses in 100 patients. Data presented as mean and upper and lower $95 \%$ Confidence intervals.

Discussion:

Although no significant differences were found, a minimal trend can be seen by just simple looking at the means of 3 groups. There is similarity between our study groups and the reference groups regarding trends. However, our grade I and grade II polyposis showed remarkable higher scoring than the reference counterparts. 